\title{
Isolated Jejunal Perforation and Mesentery Injury following a Kick on the Abdomen of a College Student: A Case Report from a District Hospital in Northern Ghana
}

\author{
Engelbert A. Nonterah $\mathbb{D}^{1},{ }^{1,2}$ Solomon Atindama, ${ }^{2}$ Emmanuel Achumbowina, ${ }^{2}$ \\ Michael B. Kaburise, ${ }^{1,2}$ Edwin Saanwie $\mathbb{D}^{2},{ }^{2}$ Aziz Ewura, ${ }^{2}$ and Majeedallahi Al-Hassan ${ }^{2}$ \\ ${ }^{1}$ Navrongo Health Research Centre, Ghana Health Service, Navrongo, Ghana \\ ${ }^{2}$ Navrongo War Memorial Hospital, Ghana Health Service, Navrongo, Ghana \\ Correspondence should be addressed to Engelbert A. Nonterah; drenanonterah@gmail.com
}

Received 8 November 2019; Accepted 7 March 2020; Published 26 March 2020

Academic Editor: Kenneth S. Waxman

Copyright (c) 2020 Engelbert A. Nonterah et al. This is an open access article distributed under the Creative Commons Attribution License, which permits unrestricted use, distribution, and reproduction in any medium, provided the original work is properly cited.

\begin{abstract}
Introduction. Isolated jejunal perforation from blunt abdominal trauma is an extremely rare intra-abdominal injury that poses a huge diagnostic challenge. Delay in diagnosis and initiation of treatment often leads to significant morbidity and mortality. Diagnosis particularly in resource-poor settings may be extremely challenging and often relies on a high index of suspicion. This is due to lack of adequate diagnostic facilities and human resource to deal with the condition with resulting high occurrence of adverse outcomes. Case Presentation. We report a case of isolated jejunal perforation with associated mesentery injury in a young college student who sustained a kick to his abdomen while playing soccer. This is an unusual presentation since most reported cases often resulted from motor vehicular accidents, bicycle handlebar, and fall from a height. We emphasized the role of critical level of suspicion with a good history and physical examination as the major source of diagnosis since diagnostic procedures, such as abdominal ultrasonography and computed tomography, are largely unavailable in most resource-constraint settings. Early surgical intervention following diagnosis leads to good recovery and reduced mortality. Conclusion. Sufficient vigilance and suspicions of small bowel perforation should always be considered after blunt trauma even when symptoms and physical findings are minimal and when diagnostic capacity is limited.
\end{abstract}

\section{Introduction}

Blunt abdominal trauma (BAT) can result in injury to intraabdominal organs, but solid organ injuries are predominant as reported in the literature [1]. Small bowel injury from $\mathrm{BAT}$ is the third most common abdominal injury $[2,3]$. Isolated perforation of the small bowel such as jejunum (isolated jejunal perforation-IJP) after blunt abdominal trauma is infrequent and extremely rare with a reported incidence of $0.3 \%[4,5]$. Diagnosis can therefore be very elusive. The commonest reported mechanisms of IJP due to BAT are motor vehicle accidents, bicycle handlebar, human and animal assault, and a few reported cases of falls from heights $[6,7]$. With many low- and middle-income countries
(LMIC) undergoing rapid urbanization and an increase in the use of motor vehicles, the incidence of IJP is likely to increase.

The mechanism of bowel perforation due to BAT is that of a sudden increase in intraluminal pressure in a fluid or air-filled bowel loop causing punctate or slit-like perforations (blowout) on the antimesenteric border of the bowel [8-10]. Absence of other intra-abdominal injuries and the associated minimal and sometimes no surrounding tissue damage leads to a huge diagnostic dilemma and significantly affects morbidity and mortality [7]. Marked improvement in the use of diagnostic peritoneal lavage (DPL), focused assessment with sonogram in trauma (FAST), and abdominal computed tomography (CT) has led to improved precision 
of diagnosis, a decrease in false-negative laparotomies, and an increase in good outcomes in abdominal injuries $[10,11]$.

Most resource-poor settings in LMIC often are bereft of modern diagnostic tools and thus rely mainly on clinical data which could be vague and physical examination which is often inconclusive in the early period of the injury [1]. Previous studies have emphasized the shortfalls in provision of adequate surgical care. Commonly identified shortfalls include lack of personnel, adequate equipment, and a huge surgical disease on few staff. Commonly available diagnostic imaging techniques such as X-rays add little diagnostic value in the initial stages as it tends to be positive only in the presence of massive pneumoperitoneum. The resultant conservative management employed with intervention only at the earliest sign of clinical deterioration tends to add significantly to adverse outcomes [12, 13].

Considering these outlined diagnostic dilemma and an unusual cause of BAT, we seek to present this case study. This case of isolated jejunal perforation occurred in a college student following a kick to the abdomen while playing soccer. In the absence of adequate diagnostic capacity in a resource-poor setting, sound clinical judgment resulted in prompt management and successful outcome. The purpose of this paper is therefore to highlight the extreme importance of critical clinical information and thorough physical examination in emergency situations.

\section{Case Presentation}

A 22-year-old male college student presented to the emergency ward with severe abdominal pain within an hour after a kick to the abdomen while playing soccer. There was no prior history of ill health before the event, and he was not known to have peptic ulcer disease. On examination, there were no external bruises on the abdomen or other associated injuries. The abdomen was not distended but full and moved with respiration. It was diffusely tender with marked tenderness at the periumbilical area. Percussion note was dull, and bowel sounds were absent. He was afebrile with a temperature of $36.9^{\circ} \mathrm{C}$. Other vital signs checked were essentially normal except for tachycardia, pulse 102 beats per minute.

An urgent chest X-ray requested showed no air under the diaphragm, while the supine abdominal X-ray was unremarkable. Focused assessment with sonogram in trauma (FAST) could not be undertaken because the hospital had no ultrasound machine at the time. Although fourquadrant tap is not encouraged in current practice as a diagnostic procedure for acute abdomen, the limited diagnostic capacity made this an option. A standard fourquadrant tap was performed which yielded bilious fluid. A preliminary diagnosis of a perforated hollow viscous was thus made and urgent exploratory laporatomy was scheduled. The patient was resuscitated with intravenous fluids (Ringer's lactate and normal saline). Broad-spectrum antibiotics (IV amoxicillin-clavulanic acid $1.2 \mathrm{~g} 8$ hourly and IV metronidazole $500 \mathrm{mg} 8$ hourly) were commenced pending surgery. Full blood count and serum electrolytes were essentially normal. The patient was resuscitated and had a successful exploratory laparotomy. The following intraoperative findings were discovered: about $1000 \mathrm{ml}$ of bilious fluid was drained from the abdomen and a single isolated jejunal perforation of about $1.5 \times 2 \mathrm{~cm}$ on the antimesenteric border (Figure 1), $10 \mathrm{~cm}$ from the ligament of Treitz, was discovered.

Two hematomas on the corresponding mesentery were present, but the mesenteric vessels were intact (Figure 2). Further exploration of the abdominal cavity showed no associated solid organ injuries. Edges of the perforation were refreshed, and primary closure of the "blowout" jejunal perforation was conducted in two layers using Vicryl 2.0 suture. Abdominal lavage with $2 \mathrm{~L}$ of warm saline was carried out. Postoperative recovery was uneventful, and the patient was discharged home on postoperative day six.

\section{Discussion}

The abdomen is the third most commonly injured part of the body following trauma either by penetrating or blunt force [14]. BAT is relatively more common and often results in solid organ (e.g., spleen and liver) rupture or damage. BAT resulting in small bowel perforation (SBP) is less rampant and results in significant morbidity and mortality when detected late. This usually occurs in the absence of less optimal diagnostic tools where expectant management is employed $[14,15]$. Over $70 \%$ of BAT is caused by motor vehicular accidents, followed by other means such as bicycle handlebar, intended and unintended human assault, fall from a height, and rarely assault from animals $[6,7,16]$. However, human assault such as a kick in the abdomen on a playing field has not been implicated in the literature as a common cause of IJP.

In the Kassena-Nankana district of Northern Ghana where War Memorial Hospital (WMH), a secondary referral facility, is located, recent mortality data show that motor vehicular accidents are becoming a leading cause of death. This trend coincides with an increase in motor and vehicular traffic and is in tandem with a rise in the socioeconomic status of the people as well as urban dwelling [17]. This trend is likely to lead to a rise in the incidence of BAT of abdominal organs including hollow viscus such as bowel injury. Mechanism of injury to small bowel in BAT is usually due to mesenteric laceration or hematomas due to direct compression between the abdominal wall and vertebral column, sheering forces, and or 'blowout' perforations due to sudden increase in intraluminal pressure in an air or fluid-filled loop of the bowel $[7,8,13,18]$. The affected part of the small bowel is usually a fixed segment such as the duodenum, duodeno-jejunal flexure [19], proximal jejunum, and terminal ileum [20], often associated with mesenteric injury [21]. More specifically, traumatic jejunal injuries occur more at the ligament of Treitz, and that is in acceleration-deceleration injuries. In the presented case, the IJP due to a blunt force from a kick to the abdomen occurred in the proximal jejunum about $10 \mathrm{~cm}$ from the ligament of Treitz.

Bowel injury from BAT poses a huge diagnostic challenge since most signs are usually vague and nonspecific; however, abdominal pain is the most commonly reported 


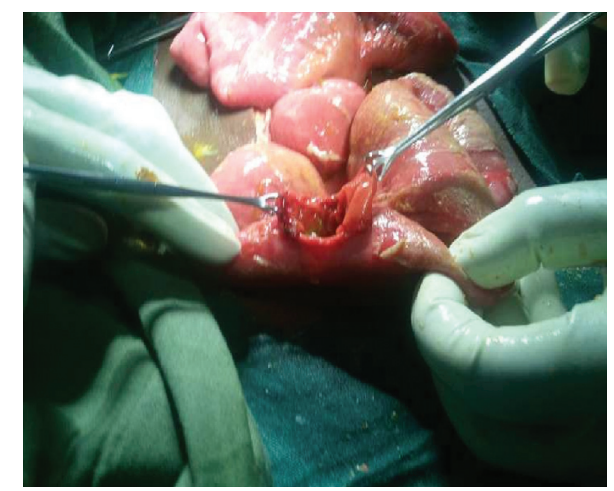

FIGURE 1: Isolated "blowout" jejunal perforation.

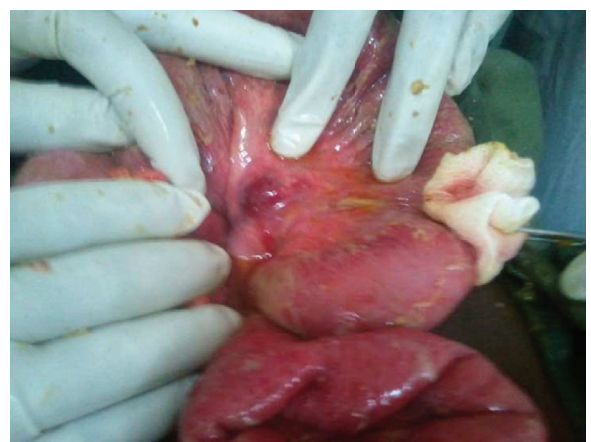

FIgURE 2: The corresponding mesenteric hematoma.

symptom together with absence of bowel sounds occurring in over $64 \%$ of cases [7] which should alert the clinician of such significant injury. Clinical presentation and physical examination alone are often not sufficient to make a diagnosis, and reliance on radiological investigations such as chest and plain abdominal X-rays (erect and supine) becomes important first-line tests in resource-constraint settings. However, in a study by Saku et al. [21] only $8 \%$ (1 out of 12 patients) of patients with bowel perforation after trauma evaluated, demonstrated free air under the diaphragm on chest X-ray as compared to $92 \%$ (11 out of 12 patients) in whom extra luminal air was detected readily by CT scan. There have also been reports that left lateral decubitus X-rays may better clarify where CXR have failed. However, this was not carried out for our patient. The use of DPL, FAST, and abdominal CT scan therefore add significantly to the precision in diagnosis and early intervention for improved outcomes [10]. Although CT scan is the gold standard for assessment of blunt trauma [7], DPL still has some diagnostic value [22-24]. Ultrasound is poor in diagnosing bowel pathologies due to presence of gas.

In the case presented, the setting had none of the above diagnostic techniques available. The available radiological investigation was of no diagnostic value as the chest X-ray showed no pneumoperitoneum, and the plain abdominal $\mathrm{X}$-rays (erect and supine) were unremarkable. This probably was due to the early presentation of the patient. If we had employed an expectant management strategy, we were more likely to pick up radiological (CXR) signs. However, we would have also increased the chances of further complications and deteriorations of the patient's health.

With surgical care increasingly being recognized as critical to the prevention and treatment of diseases, district hospitals often deprived of modern diagnostic techniques, tend to have a huge challenge in the timely diagnoses of bowel perforation. The present case illustrates the situation in a typical district hospital in Ghana, and it emphasizes that the role energetic appraisal of such cases can aid in early recognition of bowel injury for prompt surgical intervention in order to prevent complications and/or death. Building surgical capacity at district hospitals has been proposed as the most effective means to improve access to emergency and essential surgical care because these hospitals are usually the first point of care in sub-Saharan Africa (SSA) and other LMIC $[25,26]$. Evidence from the study setting and other selected hospitals across Ghana further point to the fact that medical officers spend a substantial amount of their time managing surgical and obstetrics and gynecology cases [27].

It is therefore imperative for stakeholders in healthcare delivery in resource-poor settings to concentrate on improving infrastructure in the district hospitals. It is also a call on all relevant institutions of learning to look into new and practical ways of training medical officers in the surgical disciplines to boost and improve capacity at regional and district hospitals.

\section{Conclusion}

The diagnosis of IJP is challenging, especially in resourceconstrained hospitals which are often bereft of adequate diagnostic tools. A high index of suspicion following detailed history and a thorough physical examination are essential in making prompt diagnosis. The increasing need for surgical care calls for innovative ways of building human resource capacity and infrastructure at district level facilities.

\section{Consent}

Written informed consent was obtained from the patient for publication of this case report and any accompanying images.

\section{Conflicts of Interest}

The authors declare that they have no conflicts of interest.

\section{Authors' Contributions}

EAN, SA, and EA managed the patient. EAN conceived the idea to publish the paper, designed, and coordinated the work. EAN, SA, MBK, AE, and ES collected the data and relevant patient information. EAN drafted the paper. EAN, SA, EA, MBK, AE, ES, and MA critically revised the contents of the paper. All authors read and approved the final manuscript.

\section{Acknowledgments}

The authors are very grateful to the patient for granting permission for this publication. The authors express their 
profound gratitude to Prof Jacob C. B. Dakubo (consultant surgeon at the University of Ghana Medical School, Korle Bu Teaching Hospital, Accra, Ghana) for his guidance during the development of this manuscript and for proof reading the final manuscript.

\section{References}

[1] N. Symeonidis, K. Ballas, K. Psarras et al., "Isolated small bowel perforation after blunt abdominal trauma: report of 2 cases," The Internet Journal of Surgery, vol. 27, no. 1, pp. 1-5, 2010.

[2] J. Guarino, J. M. Hassett Jr., and F. A. Luchette, "Small bowel injuries," The Journal of Trauma: Injury, Infection, and Critical Care, vol. 39, no. 6, pp. 1076-1080, 1995.

[3] G. S. Allen, F. A. Moore, C. S. Cox Jr., J. T. Wilson, J. M. Cohn, and J. H. Duke, "Hollow visceral injury and blunt trauma," The Journal of Trauma: Injury, Infection, and Critical Care, vol. 45, no. 1, pp. 69-77, 1998.

[4] D. D. Watts, S. M. Fakhry, and EAST Multi-Institutional Hollow Viscus Injury Research Group, "Incidence of hollow viscus injury in blunt trauma: an analysis from 275,557 trauma admissions from the east multi-institutional trial," The Journal of Trauma: Injury, Infection, and Critical Care, vol. 54, no. 2, pp. 289-294, 2003.

[5] K. Yusif-Zada, R. Safarov, and A. Gafarov, "Isolated small perforation following blunt abdominal trauma," Annali Italiani di Chirurgia, vol. 84, no. 6, pp. 699-703, 2013.

[6] A. Z. Sule, A. T. Kidmas, K. Awani, F. Uba, and M. Misauno, "Gastrointestinal perforation from blunt abdominal trauma," East African Medical Journal, vol. 84, no. 9, pp. 429-433, 2007.

[7] S. Goyal, S. Goyal, and M. Garg, "Single jejunal blowout perforation following blunt abdominal trauma: diagnostic dilemma," Journal of Mahatma Gandhi Institute of Medical Sciences, vol. 18, no. 2, pp. 144-146, 2013.

[8] J. V. Robbs, S. W. Moore, and S. P. Pillay, "Blunt abdominal trauma with jejunal injury: a review," The Journal of Trauma, vol. 20, no. 20, pp. 308-311, 1980.

[9] S. Deepak, "Jejunal transection after blunt abdominal trauma: a case report," International Surgery Journal, vol. 3, no. 4, pp. 2289-2291, 2016.

[10] M. Ohene-Yeboah, J. C. B. Dakubo, F. Boakye, and S. B. Naeeder, "Penetrating abdominal injuries in adults seen at two teaching hospitals in Ghana," Ghana Medical Journal, vol. 44, no. 3, pp. 103-108, 2010.

[11] M. B. Fakhree, A. Bayat, H. Farhadi, and R. Azhough, "The effect of time interval from index trauma on results of diagnostic peritoneal lavage in animal samples," Life Science Journal, vol. 10, no. 7, pp. 242-245, 2013.

[12] S. R. Thompson and A. J. A. Holland, "Perforating small bowel injuries in children: influence of time to operation on outcome," Injury, vol. 36, no. 9, pp. 1029-1033, 2005.

[13] N. A. Sandiford, R. P. Sutcliffe, and H. T. Khawaja, "Jejunal transection after blunt abdominal trauma: a report of two cases," Emergency Medicine Journal, vol. 23, no. 10, p. e55, 2006.

[14] C. Kostantinidis, V. Pitsimis, and G. Fragulidis, "Isolated Jejunal perforation following blunt abdominal trauma," Turkish Journal of Trauma \& Emergency Surgery, vol. 16, no. 1, pp. 87-89, 2010.

[15] S. M. Fakhry, M. Brownstein, D. D. Watts, C. C. Baker, and D. Oller, "Relatively short diagnostic delays ( $<8$ hours) produce morbidity and mortality in blunt small bowel injury: an analysis of time to operative intervention in 198 patients from a Multicenter Experience," The Journal of Trauma: Injury, Infection, and Critical Care, vol. 48, no. 3, pp. 408-415, 2000.

[16] N. Howes, T. Walker, N. L. Allorto, G. V. Oosthuizen, and D. L. Clarke, "Laparotomy for blunt abdominal blunt in a Civilian trauma service," South African Journal of Surgery, vol. 50, no. 2, pp. 30-32, 2012.

[17] A. Bawa, W. Paul, D. Azongo, W. George, J. F. Philips, and O. Abraham, "Road traffic fatalities-a neglected epidemic in rural northern Ghana: evidence from the Navrongo Demographic Surveillance System," Injury Epidemiology, vol. 1, no. 1, p. 22, 2014.

[18] B. V. Goudar, U. Ambi, Y. Lamani, and S. Telkar, "Isolated "blow out" jejunal perforation following blunt abdominal trauma- experience of two cases," Journal of Clinical and Diagnostic Research, vol. 5, no. 5, pp. 1120-1122, 2011.

[19] S. L. Stevens and K. I. Maull, "Small bowel injuries," Surgical Clinics of North America, vol. 70, no. 3, pp. 541-560, 1990.

[20] H. Neugebauer, E. Wallenboeck, and M. Hungerford, "Seventy cases of injuries of the small intestine caused by blunt abdominal trauma," The Journal of Trauma: Injury, Infection, and Critical Care, vol. 46, no. 1, pp. 116-121, 1999.

[21] M. Saku, K. Yoshimitsu, J. Murakami et al., "Small bowel perforation resulting from blunt abdominal trauma: interval change of radiological characteristics," Radiation Medicine, vol. 24, no. 5, pp. 358-364, 2006.

[22] P. L. Henneman, J. A. Marx, E. E. Moore, S. V. Cantrill, and L. A. Ammons, "Diagnostic peritoneal lavage," The Journal of Trauma: Injury, Infection, and Critical Care, vol. 30, no. 11, pp. 1345-1355, 1990.

[23] ATLS Subcommittee, American College of Surgeons' Committee on Trauma, and International ATLS Working Group, "Advanced trauma life support (ATLS ${ }^{\circledR}$ ): the ninth edition," Journal of Trauma and Acute Care Surgery, vol. 74, no. 5, pp. 1363-1366, 2013.

[24] M. E. Kruk, A. Wladis, N. Mbembati et al., "Human resource and funding constraints for essential surgery in district hospitals in Africa: a retrospective cross-sectional survey," PLoS Medicine, vol. 7, no. 3, Article ID e1000242, 2010.

[25] M. Galukande, J. von Schreeb, A. Wladis et al., "Essential surgery at the district hospital: a retrospective descriptive analysis in three African countries," PLoS Medicine, vol. 7, no. 3, Article ID e1000243, 2010.

[26] J. Pimenta de Castro, G. Gomes, N. Mateus, R. Escrevente, L. Pereira, and P. Jácome, "Small bowell perforation and mesentery injury after an unusual blunt abdominal traumacase report," International Journal of Surgery Case Reports, vol. 7, no. 2015, pp. 51-53, 2015.

[27] W. Mehtsun, K. Weatherspoon, L. McElrath et al., "Assessing the surgical and obstetrics-gynecology workload of medical officers; findings from 10 district hospitals in Ghana," Archives of Surgery, vol. 147, no. 6, pp. 542-548, 2012. 